\title{
Strain Mapping of Interfaces by Quantitative High-Resolution TEM
}

\author{
C.L. Johnson and Y. Zhu
}

*Center for Functional Nanomaterials, Brookhaven National Lab, Bldg. 480, Upton, NY 11973

Interfaces, common in the high-Tc superconductor $\mathrm{YBa}_{2} \mathrm{Cu}_{3} \mathrm{O}_{7-\delta}$ (YBCO), strongly influence transport properties depending on their local structure. Efforts are currently underway to correlate interface structure with superconducting properties for a series of YBCO bicrystals with various misorientation angles and degrees of $\mathrm{Ca}$ doping. In particular, $4^{\circ}$ bicrystals with $\mathrm{Ca}$ doping show enhanced transport properties (i.e., increased critical current densities) that result from an apparent reduction in the size of negatively charged regions surrounding dislocation cores [1]. This reduction in potential, which is a barrier to superconductivity, may result from compositional changes, strain, and/or bond disorder. We will present strain topographies of LAGBs and twin boundaries in YBCO determined by geometric phase analysis (GPA) of high-resolution transmission electron microscopy (HRTEM) images [2]. Recent applications of GPA to dislocations [3,4] and low-angle grain boundaries [5] revealed nanostructures not readily visible in the HRTEM images.

We employed HRTEM and GPA to map the strain and rotation associated with a $4^{\circ}$ [001] tilt boundary (Fig. 1a) in YBCO bicrystal that consists primarily of [010] dislocations (Fig. 1b) with facets that consist of paired $\langle 100\rangle$ and $<010>$ dislocations. The diagram (Fig. 1c) shows the location of the dislocations and the orientation of their Burgers vectors determined from the strain maps (Fig. 1d-h). Maps of the dilatational strains $\left(\varepsilon_{\mathrm{xx}}, \varepsilon_{\mathrm{yy}}\right.$; Fig. $\left.1 \mathrm{~b}, \mathrm{~d}, \mathrm{f}\right)$ and the shear strain $\left(\varepsilon_{\mathrm{xy}}\right.$; Fig. 1b,h) show the extension of each component into the surrounding material and the interactions among the dislocations in the boundary (e.g., in the regions surrounding grain-boundary facets). The rigid-body rotation $\left(\omega_{\mathrm{xy}}\right.$; Fig. 1e) shows the boundary morphology. Careful calibration of the microscope lens distortions allowed quantification of the strains to $<1 \%$ and rotation to $0.3^{\circ}$. Finally, the mean dilatation $\left[\Delta_{\mathrm{xy}}=\left(\varepsilon_{\mathrm{xx}}+\varepsilon_{\mathrm{yy}}\right) / 2\right.$; Fig. 1g] was used to determine the orientation of extra-half planes, which enter from the negative (i.e., compression) side of the dilatation field and terminate at the point were the dilatation changes sign (i.e., the transition to expansion). Also marked in the diagram (Fig. 1c) are two twin boundaries parallel to (110) that are not visible in the HRTEM image but appear in the dilatation and rotation maps. The twin boundaries are accompanied by small $\left(<0.5^{\circ}\right)$ rotations between the domains. Such small rotations are characteristic of O-depleted YBCO $(\delta>0.5)$.

The evolution of the strain topography with increasing misorientation angle (i.e., decreased distance between dislocations) and with $\mathrm{Ca}$ doping will be investigated. These results will be compared with information obtained from parallel studies of the same YBCO grain boundaries by electron holography, Z-contrast imaging, and quantum mechanical simulations.

[1] M.A. Schofield et al. Phys. Rev. Lett. 92 (2004) 195502-1.

[2] M.J. Hÿtch et al. Ultramicroscopy 74 (1998) 131.

[3] M.J. Hÿtch et al. Nature 423 (2003) 270.

[4] C.L.Johnson et al. Am. Mineral. 89 (2004) 1374.

[5] C.L.Johnson et al. Proc. Natl. Acad. Sci. USA 101 (2004) 17936.

[6]This work was supported by the Division of Materials Sciences, Office of Basic Energy Sciences, U.S. Department of Energy. We thank C. Jooss for providing the sample of YBCO. 

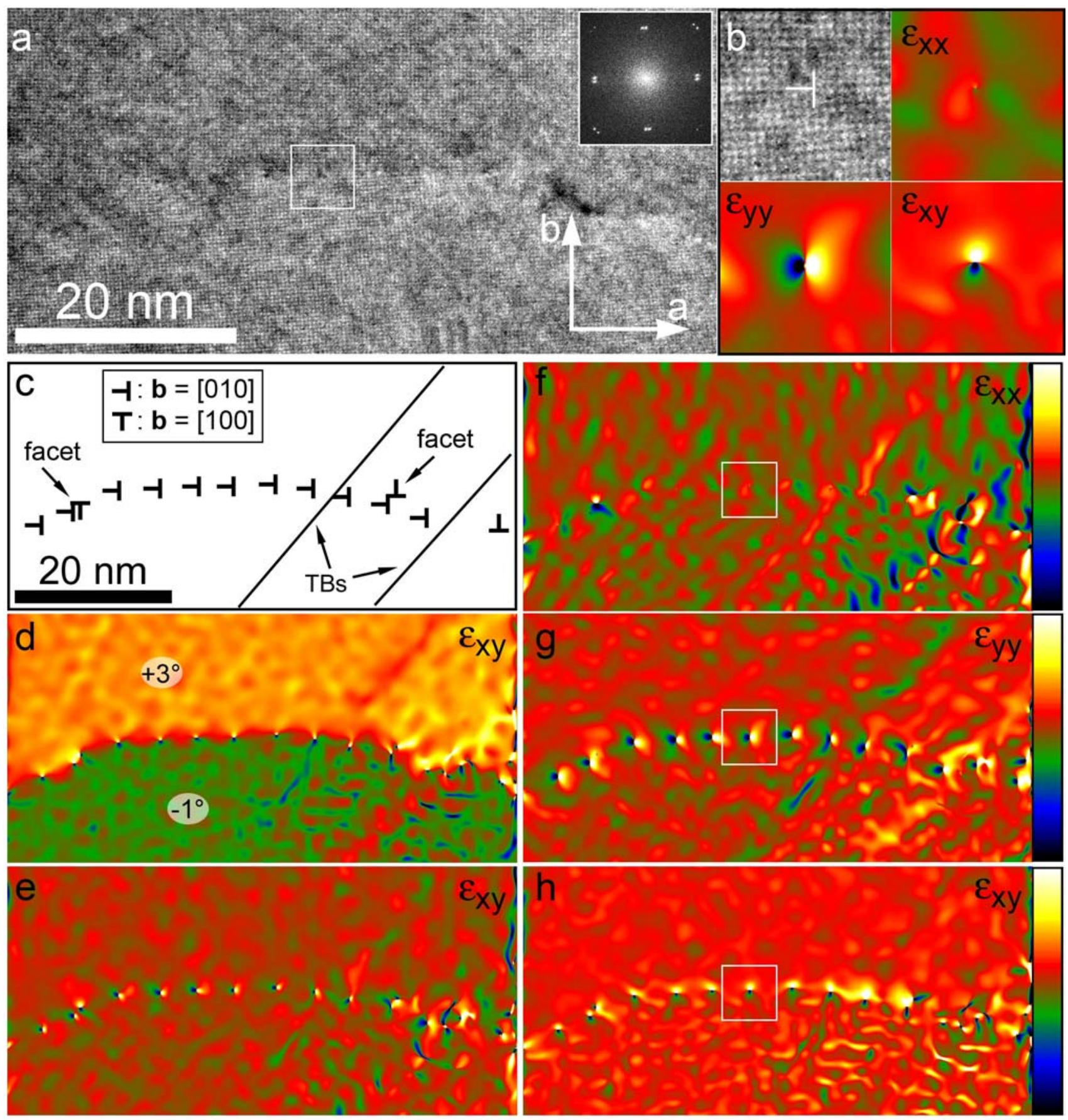

Figure 1. Strain analysis of a $4^{\circ}$ [001] tilt boundary in YBCO. (a) HRTEM image of the boundary (zone axis $=[001]$; obtained using a JEOL 4000EX TEM). The digital diffractogram (inset) shows the two sets of reflections corresponding to the two crystal orientations. The boxed region is magnified in (b), which shows a single edge dislocation and its corresponding strain fields. (c) The diagram shows the positions and orientation of the edge dislocations, grain boundary facets, and twin boundaries (TBs). Rigid-body rotation (d), mean dilatation (e), and strain (f, g, and h) maps. The boxed regions in (f), (g), and (h) are magnified in (b). The dark lobes signify regions of negative strain (i.e., compression) whereas bright lobes indicate expansion. Color scale indicates $-10 \%$ to $+10 \%$ for strain and dilatation and $-8^{\circ}$ to $+8^{\circ}$ for rotation. 